\title{
Turbine Blade Natural Frequency Estimation Using Various Methods and their Comparisons
}

\author{
Miroslav Spodniak, Jozef Novotňák*, František Heško \\ Faculty of Aeronautics, Technical University of Košice, \\ Rampová 7, 04121 Košice, Slovak Republic \\ e-mail:miroslav.spodniak@tuke.sk,jozef.novotnak@tuke.sk, \\ frantisek.hesko@tuke.sk \\ *Corresponding author
}

\begin{abstract}
The natural frequencies of aircraft jet engine parts are necessary knowledge in the design, safety and operation of the jet engine. The presented article is focused on the area of determining natural frequencies of the turbine blade of the jet engine. The article describes three different methods for determining the natural frequencies of jet engine blades. Acoustic method, method of determining natural frequencies by measuring the vibrations using an accelerometer and determination of natural frequencies by finite element method (FEM) modal analysis. The principles of each method are described in Sections 3 and 4. In Section 5, the achieved results of individual measurements are described. In the conclusion area, Section 6 of this work, the authors describe the results achieved between the various methods and their advantages/disadvantages.
\end{abstract}

Keywords: natural frequency; turbine blade; frequency measurement; vibration measurement; acoustic measurement; vibrodiagnostic

\section{Introduction}

Vibration measurement is currently used in many industries and is dealt with by vibration diagnostics, serving as a tool for modern predictive and proactive maintenance methods [1-4]. Vibrations are closely related to the technical condition of the machine, the condition of its parts and their dynamic stress [5-7]. They afford us objective information needed to determine the technical condition of the machines.

In addition to the above options, it is also possible to use vibration diagnostics to determine the natural frequencies of the blades. Natural frequencies are essential part when the stress and life of the jet engine is investigated because the resonance can lead to the failure of the jet engine. The turbine blade is one of the crucial 
parts of the engine, therefore high reliability has to be ensured during engine operation.

There are several ways to measure the natural frequency of the blade and reveal its frequency spectrum. The most commonly used methods include acoustic measurement or vibration measurement using an accelerometer [8]. In addition to these methods, however, it is possible to determine the natural frequency of the blade also on the basis of calculations.

Nowadays, there is a large number of the various methods for natural frequency estimation [9-15]. The methods can be divided into two separate categories, the first one is mentioned based on experimental methods as the mentioned measurements and second category is numerical method. When using, for example, finite element method (FEM), it is possible to carry out the numerical modal analysis and estimate natural frequencies for the turbine blade for the arbitrary number of modes. The main goal of the article is to present the results of the natural frequencies for iSTC-21v turbine blade numerically estimated and verified by the experimental results and secondly selection of the appropriate method for natural frequency estimation for the mentioned part [16]. An object of the research as mentioned is a turbine blade of iSTC-21v (Figure 1) jet engine, which is an experimental jet engine used mainly for the research purposes.

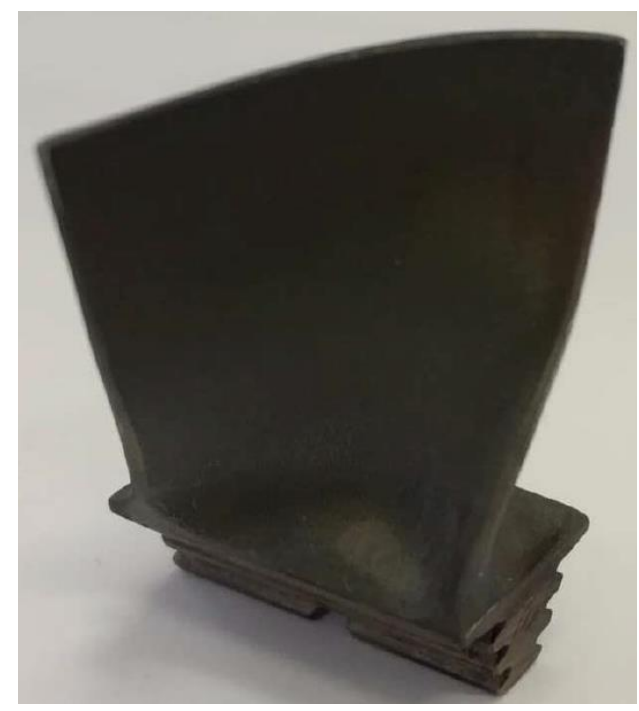

Figure 1

Turbine blade of iSTC-21v jet engine 


\section{Analysis of Vibrodiagnostic Signal}

The vibrodiagnostic signal can be analyzed in the time domain or frequency domain. The vibrations are mostly random in nature and are composed of many frequency components and can be described by amplitude and phase at a given point in time.

\subsection{Signal Analysis in the Time Domain and in the Frequency Domain}

The analysis of the vibrodiagnostic signal of the time domain (see Figure 2) is based on the evaluation of the parameters of the time courses of the signals. In the time domain, it is easy to evaluate the instantaneous, mean and effective values of the signal or signal envelope. In the case of the predominant random component of the signal (so-called random vibration), selected statistical calculations can be applied for analysis. Time analysis is suitable if there is a single or at least dominant source of vibration, or otherwise there is a loss of diagnostic information in the signal noise caused by the transmission of vibrations from different areas of the machine complex and the possibility of locating the cause of vibration is very limited.
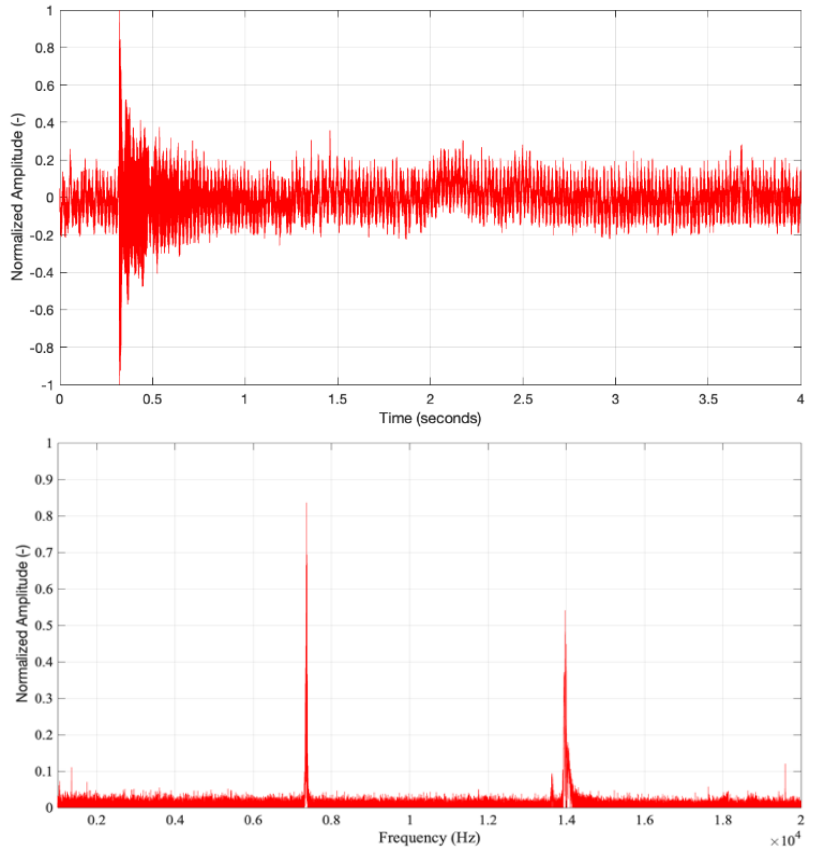

Figure 2

Measured signal in the time domain (top) and in the frequency domain (bottom) 
Frequency analysis eliminates the disadvantages of time domain. The aim of spectral analysis is to describe the distribution of signal components in the frequency domain (see Figure 2), to express the analyzed signal using orthogonal basis functions.

A Discrete Fourier Transformation (DFT) can be used to determine the frequency components of the signal. The DFT calculates finite sequences in the time and frequency domains. Several fast algorithms are available for DFT calculation.

Fast Fourier Transformation (FFT) is a typical representative of a fast algorithm for DFT calculation [14]. For a complex input sequence (this is the most general case, the input sequence can also be purely real)

$$
x(n)=x_{r}(n)+j x_{i}(n) \quad n=0,1, \ldots, N-1
$$

the FFT algorithm is defined by the equation

$$
X(k)=X_{r}(k)+j X_{i}(k)=\sum_{n=0}^{N-1} x(n) e^{-j \frac{2 \pi k n}{N}} \quad k=0,1, \ldots, N-1
$$

where, $N$ is the number of samples. For inverse FFT (IFFT) we can use equation:

$$
x(n)=x_{r}(n)+j x(n)=\frac{1}{N} \sum_{k=0}^{N-1} X(k) e^{j 2 \pi \frac{k n}{N}} \quad n=0,1, \ldots, N-1
$$

All FFT calculations assume a linear system. The length of the classical FFT calculation depends on the length of the input signal, which is the shortest for the lengths of powers of 2. The FFT calculates the individual frequency components of the measured time complex signal, according to predetermined frequency range and resolution requirements. The number of values of the frequency spectrum is halved with respect to the number of values of the time signal, where $f_{\max }$ is equal to half of the sampling frequency $f_{v z}=1 / \Delta t$. This is related to Shannon's sampling theorem, according to which the sampling frequency must be at least twice as high (Nyquist frequency) as the frequency of the highest harmonic component contained in the measured signal. 


\section{Acoustic Frequency Measurement}

We used a condenser microphone for acoustic measurement of the blade frequency. The frequency range of the microphone is from $40 \mathrm{~Hz}$ to $20 \mathrm{kHz}$ $( \pm 5 \mathrm{~dB})$. The signal was sampled with a resolution of 24 bits and a sampling frequency of $48 \mathrm{kHz}$. The measurement process is shown in the Figure 4. The experimental setup of frequency measurement using a microphone is shown in Figure 8.

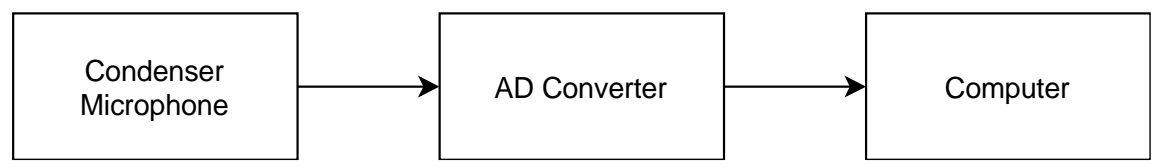

Figure 4

Block diagram of acoustic frequency measurement

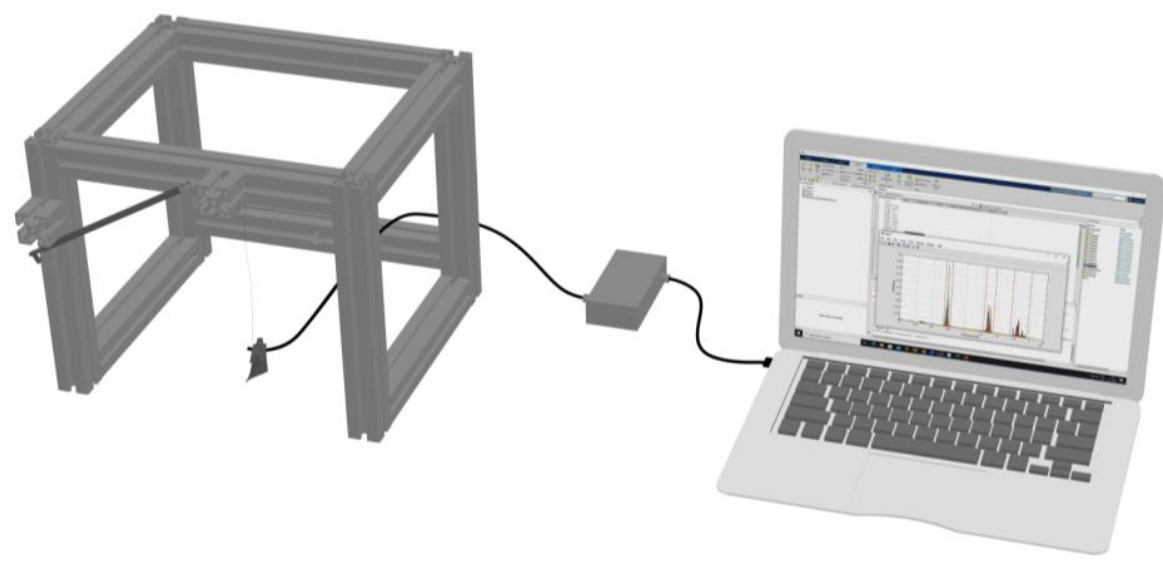

Figure 5

Experimental setup of frequency measurement using a microphone

\section{Frequency Measurement Using a Piezoelectric Accelerometer}

A piezoelectric accelerometer KD-37 was used to measure the frequency. Its use is suitable, especially in terms of a wide frequency range. The accelerometer generates an electric charge at its output, but its value is very low and it was necessary to amplify it. A charge amplifier was designed (see Figure 6) to amplify the signal, which was adapted exactly for this type of accelerometer, due to its frequency response and electrical parameters (see Table 1). 
Table 1

KD-37 Accelerometer parameters

\begin{tabular}{|l|l|l|}
\hline Parameter & Value & Units \\
\hline Charge Sensitivity & 60 & $\mathrm{pC} / \mathrm{g}$ \\
\hline Linear frequency range $( \pm 3 \mathrm{~dB})$ & 15000 & $\mathrm{~Hz}$ \\
\hline Sensor Capacity & 0.61 & $\mathrm{nF}$ \\
\hline Cable Capacity 1.5 Meters Long & 0.15 & $\mathrm{nF}$ \\
\hline Mass of the Accelerometer & 45 & $\mathrm{grams}$ \\
\hline
\end{tabular}

Given the above parameters the capacitance of the $\mathrm{C} 1$ capacitor found in the feedback of the charge amplifier is set to $0.76 \mathrm{nF}$ to obtain a charge amplifier gain of $1 \mathrm{mV} / \mathrm{pC}$. Resistor R1 affect the lower limit frequency and resistor R2 affect the upper limit frequency [18-20]. These frequency limits can be defined with respect to the use of the sensor (for example, the assumed frequency measurement band), but it is also necessary to take into account the sensor parameters (frequency range for example). TL071 was used as an operational amplifier.

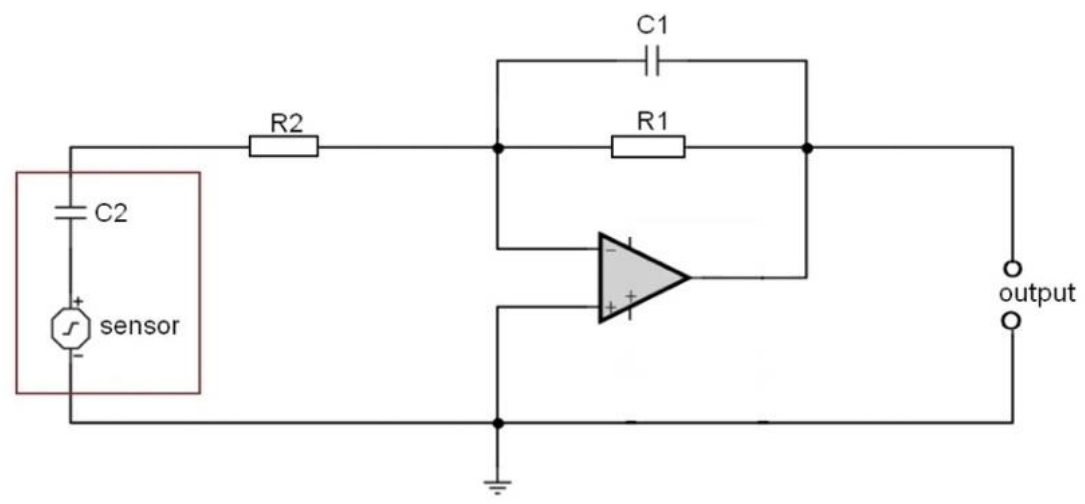

Figure 6

Circuit diagram of a charge amplifier

The measurement process consists of two steps. The first step is to measure the frequency and obtain the necessary data. The second step is the processing and evaluation of the measured data using MATLAB software.

The basic element of frequency measurement is a piezoelectric accelerometer. The output of the accelerometer is fed to the input of the charge amplifier, where the signal is adjusted and amplified. After amplification, the signal is fed to an $\mathrm{A} / \mathrm{D}$ converter, where it is sampled at a frequency of $48 \mathrm{kHz}$ with a resolution of 24 bits and converted into digital form. After this process, the signal is sent to the computer, where it is recorded and ready for further processing. The whole measurement process is described by a block diagram in Figure 7. Photograph of the experimental setup of frequency measurement using an accelerometer is shown in Figure 8. 


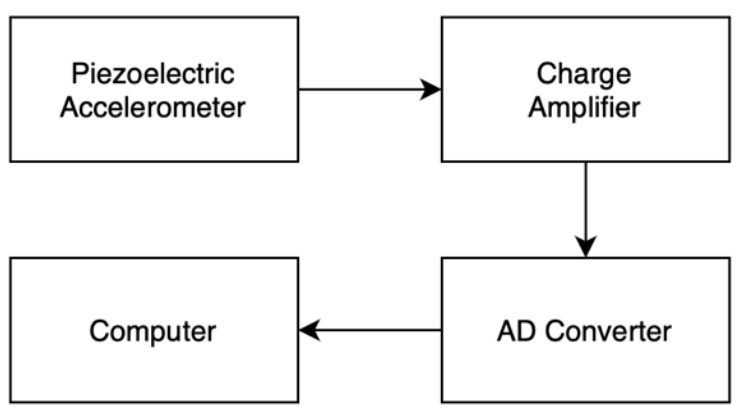

Figure 7

Block diagram of frequency measurement

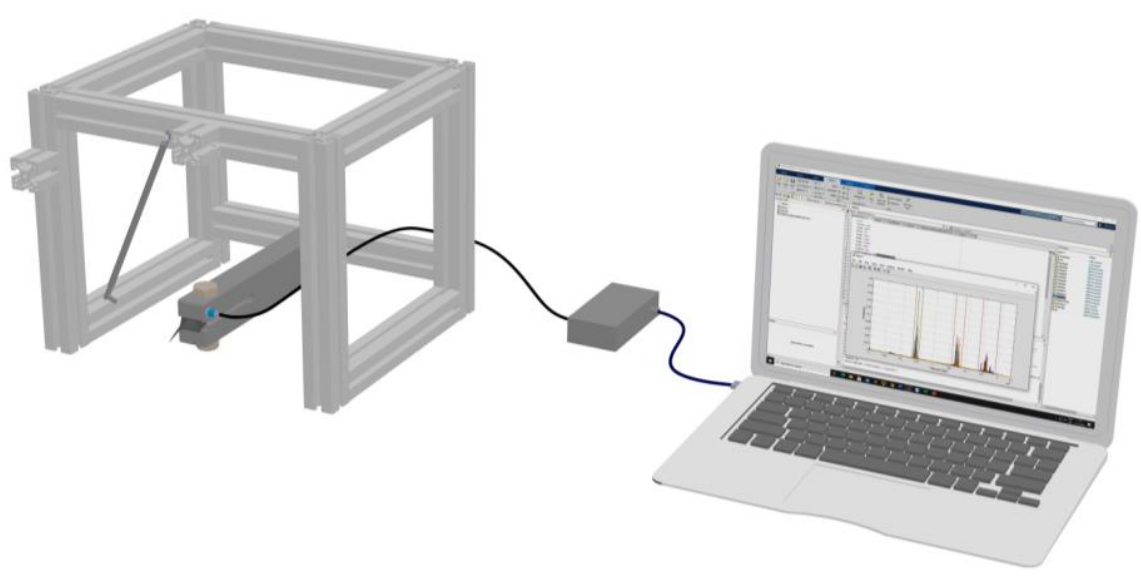

Figure 8

Experimental setup of frequency measurement using an accelerometer

\section{Results of Spectral Analysis of the Measured Signal}

For data processing of the measured signal, we used MATLAB. Data must be converted from the time domain to the frequency domain. The fft function [17] was used to detect individual frequencies in the measured signal.

The results obtained by the calculation method were compared with the results of acoustic frequency measurement and with the results of frequency measurement with an accelerometer.

In order to ensure a safe operation, range for jet engine the Campbel diagram is constructed and for the creation of such a diagram, the resonant frequencies are 
essential part, therefore the research is focused on resonant frequencies and peaks that occurs in the following Figures. Oftentimes, first three modes are important for the Campbel diagram creation. In the proposed article, the first three turbine blade modes for the iSTC-21v jet engine are investigated.

As it was mentioned in previous chapters, natural frequencies are experimentally measured using two methods, or two different sensors, thus acoustic sensor and the accelerometer. The third method for the natural frequency estimation is devoted to the finite element modelling approach and natural frequencies for the turbine blade are computed.

\subsection{Acoustic Results}

The results of the first mentioned method are presented in Figure 9, where the dependency between the frequency and its amplitude can be seen. A total of 11 turbine blades of the iSTC- $21 \mathrm{v}$ jet engine were investigated and the measurement for each blade was repeated 5 times. During the experiment each blade was loosely attached using the nylon cord (see Figure 5) and the frequency was excited using mechanical hammer. The first resonant mode of the blade is in a range 6800 $-7500 \mathrm{~Hz}$, a second mode is $13500-14500 \mathrm{~Hz}$ and the third one is in a frequency range $17000-20000 \mathrm{~Hz}$.

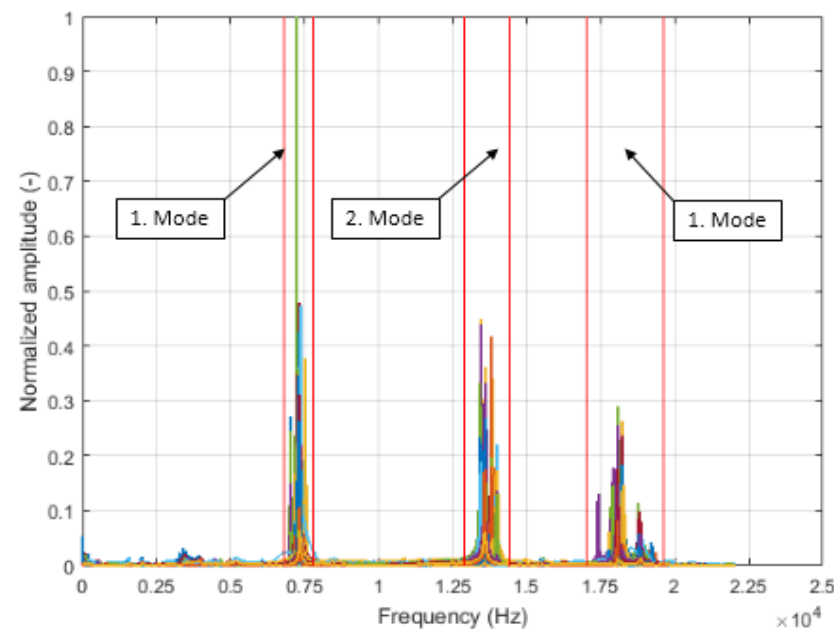

Figure 9

Measured frequency signal for turbine blade using acoustic method 


\subsection{Accelerometer Method Results}

The accelerometer was used for the second experiment, the same number of turbine blade (11) of the iSTC-21v jet engine was measured five times repeatedly for each blade, to ensure the measurement correctness. The excitation was performed similarly like during the acoustic experiment, however, the blade was attached in the clamp to which the accelerometer was attached (see Figure 8). The results of the accelerometer method are presented in Figure 10. When we compare the results with the acoustic measurement, similarity between the two methods is undoubtedly proved. Also, the first resonant mode of the blade is in a same range 6800-7500 $\mathrm{Hz}$, second mode has the range $13500-14500 \mathrm{~Hz}$ and the third one is in a frequency range 17500-20000 Hz.

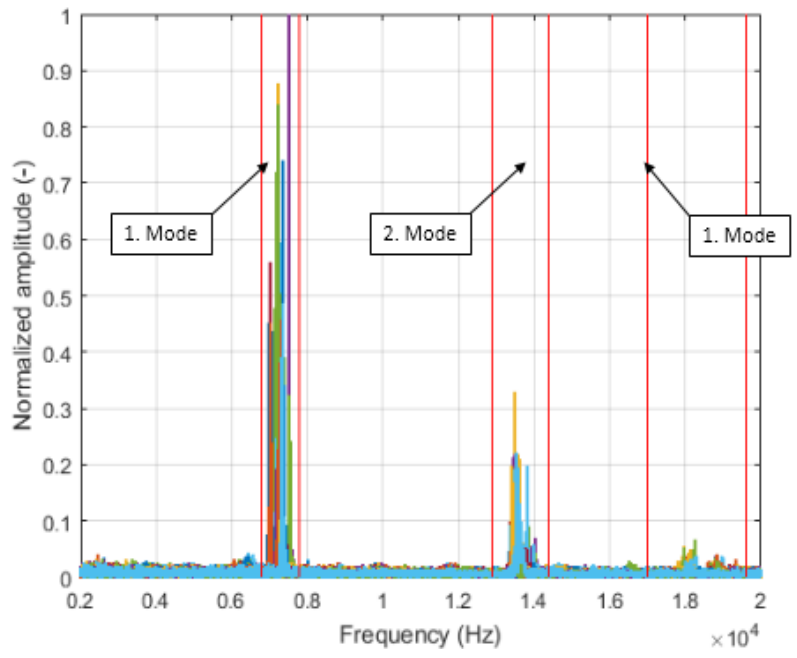

Figure 10

Measured frequency signal for turbine blade using accelerometer

\subsection{FEM Modal Analysis Results}

The first step when the FEM modal analysis is performed is to create the geometry of an investigated object, in order to perform a modal analysis, the geometry of the turbine blade was created and is described in the article [9]. However, the geometry in the leading edge area was not completely matching the real geometry, therefore for this study the geometry and mesh was updated and the final mesh is shown in Figure 11. The old and updated geometry is shown in Figure 11, the old one is in the left and new updated geometry of the turbine blade is in the right part of the Figure 11. Modal analysis is perfomed in the ANSYS APDL software. The number of finite elements is 368591 and the element type is SOLID 95 hexa elements, the 
frequency range for the modal analysis is set from $0-24 \mathrm{kHz}$. The material of the blade is ZS6k alloy. The nodes sets in the fir tree root area are created for the boundary conditions application. Translation in $\mathrm{x}, \mathrm{y}$ and $\mathrm{z}$ direction is fixed. Such a boundary condition is simulating the real mounting conditions in turbine disc during the engine operation. The results of the FEM modal analysis are presented in the following figures, where are computed the shapes and natural frequencies.
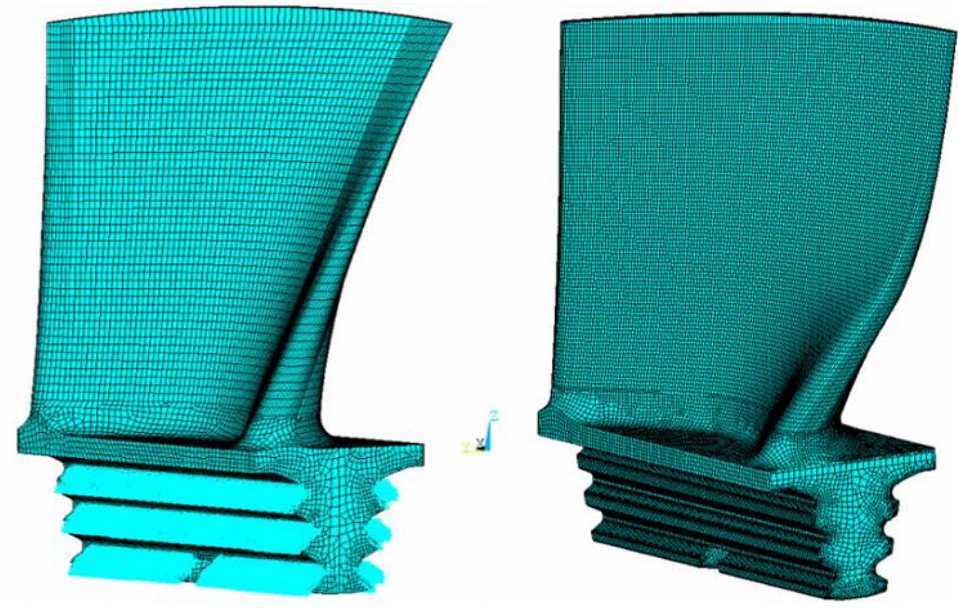

Figure 11

Finite element model for modal analysis in ANSYS APDL

The first mode is presented in Figure 12, natural frequency has a value $7524.2 \mathrm{~Hz}$.

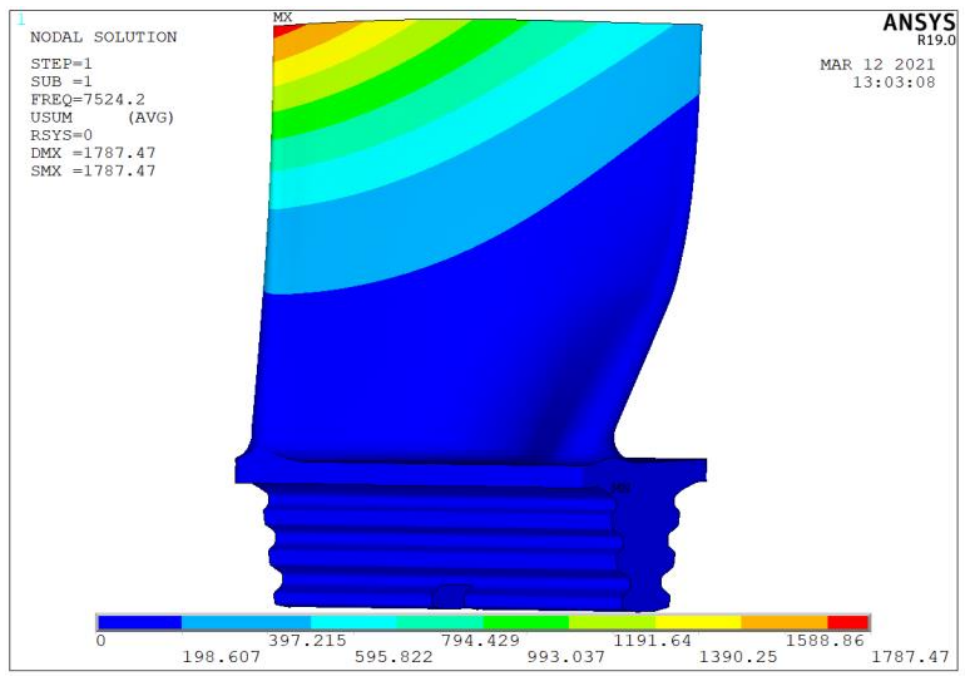

Figure 12

First natural frequency estimated in ANSYS APDL 
Second resonant mode for turbine blade is presented in the Figure 13, where we can see the shape for second natural frequency, which is $13377.8 \mathrm{~Hz}$. The maximal deformation of the turbine blade for the first mode is mainly concentrated in the trailing are at the tip of the blade, however, the deformations for the second mode are concentrated in the leading edge are also at the tip of the blade.

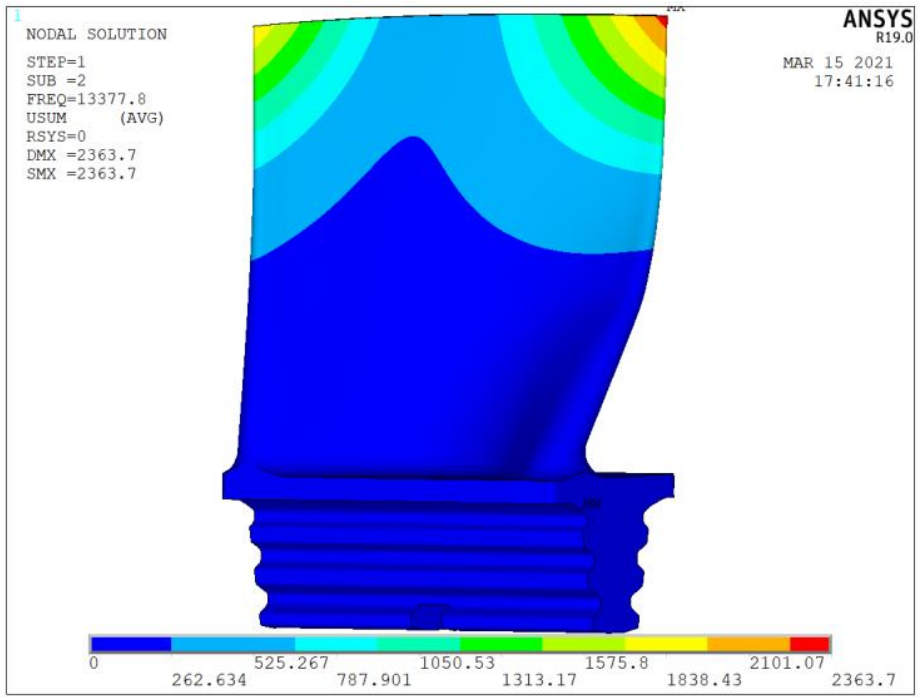

Figure 13

Second natural frequency estimated in ANSYS APDL

As it can be seen in Figures 12 and 13 the natural frequency for both modes is in the same ranges as during the experiment. Third natural frequency estimated using modal analysis in ANSYS APDL has a value $19261.4 \mathrm{~Hz}$ and the deformation during this resonant mode are shown in Figure 14. The character of the deformation is in comparison with previous two modes slightly different, the maximal deformation is concentrated in the middle of the blade chord at the tip as can be seen in Figure 14.

An estimated frequencies using FEM method and frequencies measured by mentioned methods are presented in the Table 2. 


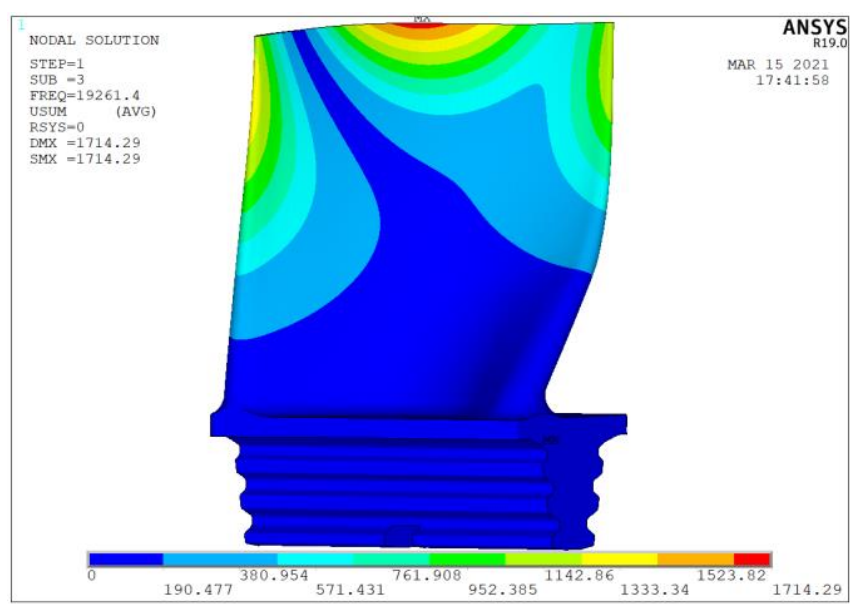

Figure 14

Third natural frequency estimated in ANSYS APDL

Table 2

Turbine blade natural frequencies resume

\begin{tabular}{|c|c|c|c|c|}
\hline Method & Measurement & 1. Mode [Hz] & 2. Mode [Hz] & 3.Mode [Hz] \\
\hline \multirow{4}{*}{ Acoustic } & 1 & 7523 & 13872 & 18229 \\
\cline { 2 - 5 } & 2 & 7531 & 13864 & 18238 \\
\cline { 2 - 5 } & 3 & 7523 & 13864 & 18213 \\
\cline { 2 - 5 } & 4 & 7531 & 13880 & 18238 \\
\cline { 2 - 5 } & 5 & 7523 & 13872 & 18229 \\
\hline Acoustic Aver. & & 7526.2 & 13870.4 & 18229.4 \\
\hline \multirow{3}{*}{ Accelerometer } & 1 & 7528 & 13860 & 18250 \\
\cline { 2 - 5 } & 2 & 7527 & 13850 & 18245 \\
\cline { 2 - 5 } & 3 & 7528 & 13855 & 18255 \\
\cline { 2 - 5 } & 4 & 7528 & 13880 & 18260 \\
\hline Accel. Aver. & & 7527 & 13860 & 18248 \\
\hline FEM & 1 & 7527.6 & 13861.0 & 18251.6 \\
\hline
\end{tabular}

Apart from the measured frequencies in Table 2, there are also described averages for particular measurements. An average of the acoustic measurement is 7526.2 $\mathrm{Hz}$ for the first mode, which is $0.045 \%$ higher than the estimated value of the FEM analysis. The second acoustically measured mode is $3.6 \%$ higher than calculated frequency and the third one is $5.24 \%$ lower value than the frequency calculated by the FEM method. The accelerometer average frequency of the first mode is 7527.6 , this value is $0.27 \%$ higher than the natural frequency estimated by FEM. The frequency of the second mode is $3.68 \%$ higher in comparison with 
FEM and the third one is $5.36 \%$ lower value than the frequency calculated by the FEM method.

\section{Conclusions}

The aim of the article was to compare different methods for determining the natural frequencies of jet engine blades. To determine the frequency, we compared the acoustic method, the measurement method using an accelerometer and FEM modal analysis. When determining the frequency using an accelerometer, we designed our own charge amplifier, adapted to the accelerometer we used.

The results of acoustic and accelerometer measurements were approximately the same in all 3 modes. Compared to the FEM method, we achieved similar results in mode 1 for all 3 measurement methods. In mode 2 and mode 3, the results were slightly different from the FEM method. This can be due to the geometry of the blade and the fact that the blade has already been used in the jet engine. In order to be able to more accurately predict the natural frequencies for modes 2 and 3, it would be necessary to obtain the geometry of the blade already used and include blade wear, which is, however, more time consuming than acoustic or accelerometer measurement.

Comparative measurements have confirmed that the natural frequency measurement can be solved in several ways. With the FEM method, however, it is necessary to create an accurate geometric model of the measured blade and to include blade wear which can be time consuming. Otherwise, if we create only a general blade model that does not involve wearing, then the results at higher frequencies will be inaccurate, which was also confirmed in the above measurements. For acoustic measurement and measurement using an accelerometer, we do not need to solve the geometry of the blade and the determination of the natural frequency can be done faster, but it is necessary to have the necessary equipment. However, the measurement shows that all three methods are applicable and with the correct geometric model, the results are similar.

The issue of determining the natural frequencies of the blades has a wide scope. The natural frequency of the blades will also change, with respect to the mechanical condition of the blade and with respect to the ambient temperature, which could be the subject of further research in the given issue. Further research could determine the trend of the change in the natural frequency of the blade with respect to the ambient temperature, which is also important in terms of safety, in the operation jet engines.

\section{Acknowledgement}

This research was funded by the Slovak Research and Development Agency, grant number APVV-17-0184 and grant number APVV-18-0248 and the Research Agency, ITMS code number 313011T557. 


\section{References}

[1] A. R. Bastami, S. Vahid: "A Comprehensive Evaluation of the Effect of Detect Size in Rolling Element Bearings on the Statistical Features of the Vibration Signal", in Mechanical Systems and Signal Processing, Vol. 151, 2020

[2] T. A. Shifat, J. W. Hurt: “An Effective Stator Fault Diagnosis Framework of BLDC Motor Based on Vibration and Current Signals", in IEEE Access, Vol. 8, 2020

[3] M. M. Jovanovič: "Detection of Jet Engine Viper Mk 22-8 Failure in Vibration Spectra", In: Mitrovic N., Mladenovic G., Mitrovic A. (eds) Experimental and Computational Investigations in Engineering. CNNTech 2020, Lecture Notes in Networks and Systems, Vol. 153, Springer, Cham.

[4] A. S. Komshin and V. I. Pronyakin: "Modern Diagnostic of Aircraft Gas Turbine Engines”, in IOP Conf. Ser.: Mater. Sci. Eng., Vol. 714, 2020

[5] G. Manhertz, A. Bereczky: "STFT Spectrogram Based Hybrid Evaliation Method for Rotating Machine Transient Vibration Analysis", in Mechanical Systems and Signal Processing, Vol. 154, 2021

[6] S. Fabry, M. Ceskovic: "Aircraft Gas Turbine Engine Vibration Diagnostic”, Magazine of Aviation Development, Vol. 5, 2017, pp. 24-28

[7] Tian Lv and Y. Zhang: "Dynamic Stress Analysis for Vibratory Stress Relief Through the Vibration Platform," 2014 IEEE Workshop on Electronics, Computer and Applications, Ottawa, ON, Canada, 2014, pp. 560-563, doi: 10.1109/IWECA.2014.6845682

[8] Naim Baydar, Andrew Ball: "A Comparative Study of Acoustic and Vibration Signal in Detection of Gear Failures Using Wigner-Ville Distribution," in Mechanical Systems and Signal Processing, Vol. 15, Issue 6, 2001, pp. 1097-1107, doi: 10.1006/mssp.2000.1338

[9] Spodniak, M., Semrád, K., Főző, L., Pavlinský, J.: "FEM Analysis of Natural Frequencies of Jet Engine iSTC-21v Turbine Blade," in SAMI 2019 - IEEE $17^{\text {th }}$ World Symposium on Applied Machine Intelligence and Informatics, Proceedings, January 2019, Article number 8782781, 2019, pp. 287-292

[10] Pridorozhnyi, R. P., Zinkovskii, A. P., Merkulov, V. M. et al: "Calculationand-Experimental Investigation on Natural Frequencies and Oscillation Modes of Pairwise-Shrouded Cooled Turbine Blades," Strength Mater 51, 2019, pp. 817-827

[11] Gareth L. Forbes, Robert B. Randall: "Estimation of turbine blade natural frequencies from casing pressure and vibration measurements," in Mechanical Systems and Signal Processing, Volume 36, Issue 2, 2013, pp. 549-561 
[12] Gillich Gilbert-Rainer, Mituletu Ion Cornel, Nedelcu Dorian, Hamat Codruta Oana: "A procedure for an Accurate Estimation of the Natural Frequencies of Structures," in Vibroengineering PROCEDIA, Vol. 19, 2018, pp. 123-128

[13] M. N. Chekardovskiy, S. M. Chekardovskiy, A. A. Rozboynikov and T. G. Ponomareva: "A Frequency Model of Vibrational Processes in Gas-Turbine Drives of Compressor Stations of Main Gas Pipelines“, in IOP Conf. Ser.: Mater. Sci. Eng., Vol. 154, 2016

[14] Yang, Yuan-Jian \& Yang, Liang \& Wang, Hai-Kun \& Zhu, Shun-Peng \& Huang, Hong-Zhong,: "Finite Element Analysis for Turbine Blades with Contact Problems, “ in International Journal of Turbo \& Jet-Engines, 2016, pp. 367-371

[15] Poulose, P., Hu, Z.,: "Strength Evaluation and Failure Prediction of a Composite Wind Turbine Blade Using Finite Element Analysis," in Proceedings of the ASME 2010 International Mechanical Engineering Congress and Exposition, Vol. 3, 2010, pp. 295-301

[16] Beneda, K., Andoga, R., Andoga, Főző, L., "Linear Mathematical Model for State-Space Representation of Small Scale Turbojet Engine with Variable Exhaust Nozzle," in Periodica Polytechnica Transportation Engineering, Vol. 46, No. 1, pp. 1-10, 2018

[17] T. Harčarik, J. Bocko and K. Masláková: "Frequency Analysis of Acoustic Signal using the Fast Fourier Transformation in MATLAB“, in Procedia Engineering, Vol. 48, 2012, pp. 199-204

[18] J. Novotňák, M. Šmelko and M. Fil'ko: "The Design of the Engine Control Unit of Small Aircraft Engine", in Acta Avionica: journal of science, Vol. 20, No. 2, Košice (Slovensko): Faculty of Aeronautics, 2018, pp. 29-37

[19] M. Kellet: "Charge Amplifiers for Piezo Electric Accelerometers" [Online] Available on the Internet: 〈http://www.mkesc.co.uk/Chargeamps.pdf>

[20] J. Karki: "Signal Conditioning Piezoelectric Sensors “ [Online] Available on the Internet: 〈http://www.ti.com/lit/an/sloa033a/sloa033a.pdf> 